University of Nebraska - Lincoln

DigitalCommons@University of Nebraska - Lincoln

$4-8-2004$

\title{
Hybrid atomistic-coarse-grained treatment of thin-film lubrication.
} I

Z.-B. Wu

University of Nebraska-Lincoln

Dennis J. Diestler

University of Nebraska-Lincoln, ddiestler1@unl.edu

Ruqiang Feng

University of Nebraska-Lincoln, rfeng1@unl.edu

Xiao Cheng Zeng

University of Nebraska-Lincoln, xzeng1@unl.edu

Follow this and additional works at: https://digitalcommons.unl.edu/chemzeng

Part of the Chemistry Commons

Wu, Z.-B.; Diestler, Dennis J.; Feng, Ruqiang; and Zeng, Xiao Cheng, "Hybrid atomistic-coarse-grained treatment of thin-film lubrication. I" (2004). Xiao Cheng Zeng Publications. 28.

https://digitalcommons.unl.edu/chemzeng/28

This Article is brought to you for free and open access by the Published Research - Department of Chemistry at DigitalCommons@University of Nebraska - Lincoln. It has been accepted for inclusion in Xiao Cheng Zeng Publications by an authorized administrator of DigitalCommons@University of Nebraska - Lincoln. 


\title{
Hybrid atomistic-coarse-grained treatment of thin-film lubrication. I
}

\author{
Z.-B. Wu \\ Department of Chemistry, University of Nebraska-Lincoln, Lincoln, Nebraska 68588 \\ and LNM, Institute of Mechanics, Academia Sinica, Beijing 100080, China \\ D. J. Diestler \\ Department of Agronomy and Horticulture, University of Nebraska-Lincoln, Lincoln, Nebraska 68583 \\ R. Feng \\ Department of Engineering Mechanics, University of Nebraska-Lincoln, Lincoln, Nebraska 68588 \\ X. C. Zeng a) \\ Department of Chemistry, University of Nebraska-Lincoln, Lincoln, Nebraska 68588
}

(Received 10 December 2003; accepted 14 January 2004)

\begin{abstract}
A technique that melds an atomistic description of the interfacial region with a coarse-grained description of the far regions of the solid substrates is presented and applied to a two-dimensional model contact consisting of planar solid substrates separated by a monolayer fluid film. The hybrid method yields results in excellent agreement with the "exact" (i.e., fully atomistic) results. The importance of a proper accounting for the elastic response of the substrates, which is reliably and efficiently accomplished through coarse-graining of the far regions, is demonstrated. (C) 2004 American Institute of Physics. [DOI: 10.1063/1.1667474]
\end{abstract}

\section{INTRODUCTION}

Thin-film lubrication involves the relative sliding of two solid substrates separated by a thin fluid film (e.g., lubricant or adsorbed impurity). ${ }^{1}$ At the interface the substrates make molecular contact at relatively few asperities (irregular prominences of microscopic dimensions), through which they interact via purely mechanical processes such as elastic distortion, plastic flow of the asperities and flow of lubricant, ${ }^{2}$ as well as by physiochemical processes such as adsorption, ${ }^{2}$ adhesion, ${ }^{2,3}$ and chemical reaction. ${ }^{4}$ Hence, thinfilm lubrication entails a complicated interplay among various processes that couple spatial and temporal scales spanning the gamut between molecular and macroscopic.

That coupling occurs at the interface, where fluid molecules, as well as neighboring solid atoms of the substrates, can undergo large-amplitude (diffusive) motions. The details of both fluid and solid molecular motions in the vicinity of the interface are expected to influence strongly the frictional force (i.e., the minimum force needed to initiate, or to maintain, sliding). We take it for granted that the fluid film and the "near" regions of the solid substrates (i.e., the several layers of solid that border the interface) must be described atomistically in order to predict reliably thermomechanical properties. The question is: How extensive must the near regions be? It is clearly impracticable to treat realistic (macroscopic) tribological systems wholly at the molecular scale, although computer simulation of single, idealized nanoscopic asperities is feasible. ${ }^{5-7}$ Moreover, since atoms in regions of the solid far removed from the interface (i.e., the "far" regions) execute only small oscillations about their equilibrium positions, the far regions behave as (linear) elastic solid. This

a)Electronic mail: xzeng1@unl.edu suggests that we may be able to "coarse-grain" the far regions, treating them at the continuum (macroscopic) scale and thereby submerging the details of atomic motion, yet still having a reliable description of the process of interest. The next question is: How do we merge the coarse-grained treatment adequate for the far regions with the atomistic description necessary for the fluid and near regions in order to achieve a self-consistent reliable global description?

Coarse-graining refers in general to a procedure that eliminates atomic degrees of freedom to achieve a reduced description of the system in terms of fewer degrees of freedom, which pertain to quasi-particles interacting via an effective potential energy that incorporates (approximately) the influence of the "lost" atoms. Coarse-graining has been applied to polymer melts, ${ }^{8-10}$ materials ${ }^{11-15}$ and biological structures. ${ }^{15}$ Here we adapt the "quasi-continuum approach" 12 to the description of sliding at a fluid-solid interface.

The purpose of this paper is twofold: (1) to present a new hybrid atomistic-coarse-grained Monte Carlo method for handling reversible multiscale processes at fluid-solid interfaces; (2) to demonstrate the importance of reliably modeling the elastic response of the far regions in determining the shear stress.

The key idea behind the hybrid approach is to coarsegrain the system partially. The far regions of the substrates, which behave essentially as elastic solid, are covered by a coarse-graining (CG) mesh, whose nodes coincide with relatively few atoms. The coarse-grained regions are dynamically constrained according to the quasicontinuum procedure of Tadmor et al.: ${ }^{12,16}$ When a CG element is distorted by displacement of its nodes, the underlying lattice is assumed to be deformed according to the same interpolations as those used in the finite-element method. ${ }^{17}$ Hence, all atoms on the 


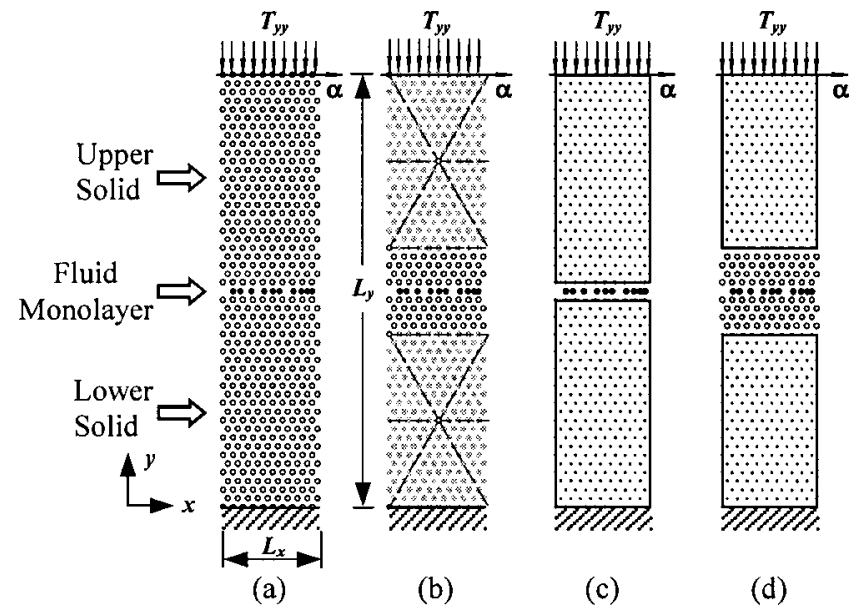

FIG. 1. Schematic of idealized two-dimensional contact. (a) Fully atomistic description; (b) partial coarse-graining of far regions of substrates; (c) wholly rigid substrates; (d) rigid far regions. All atoms are depicted in their initial configuration at $\alpha=0$.

underlying lattice are displaced in unison. This constraint greatly reduces the number of degrees of freedom of the system, replacing the original atoms of the far regions with the nodes of the CG mesh. For the constant-strain elemental deformations assumed in this work the coordinates of the underlying atoms are related to the coordinates of the nodes by a linear transformation, which can be used to derive a coarse-grained Hamiltonian that regards the nodes as quasiparticles. ${ }^{18}$ The effective interactions among the quasiparticles are mediated by the underlying atoms constrained to move in lockstep with the nodes. The Hamiltonian of the whole system consists of the effective-particle Hamiltonian for the far regions plus the original real-particle Hamiltonian for the film and near regions. The near and far regions are coupled by interactions between near-region solid atoms and atoms underlying the adjacent CG elements. This effective total Hamiltonian can be used in Monte Carlo computer simulation of reversible processes (e.g., sliding).

In order to investigate the importance of a proper modeling of the elastic response of the far regions in the context of tribology, we simulated reversible sliding at a single model contact: Two-dimensional (2D) planar substrates separated by a monolayer film. We examined several dynamical approximations for the response of the far regions, comparing the approximate results with the "exact" results obtained from the fully atomistic simulation.

\section{MODEL TRIBOLOGICAL SYSTEM}

The idealized 2D contact consists of two identical crystalline substrates separated by a thin fluid film at an atomically flat interface [see Fig. 1(a)]. The top layer of atoms of the upper substrate (top wall) and the bottom layer of the lower substrate (bottom wall) are taken to be rigid with the nearest-neighbor distance fixed at the lattice constant $a$ of the hexagonal close-packed structure. The bottom wall is assumed to remain fixed in the "laboratory" reference frame; the top wall can be translated in the $x$ and $y$ directions, but is assumed to remain parallel with the bottom wall. Thus, the walls function as handles by which the substrates can be moved relative to each other. They are technically not part of the system, which comprises the intervening (active) solid substrates plus the fluid film.

The system is bounded by an $L_{x} \times L_{y}$ rectangle, where $L_{y}$ is the distance between the rigid planes of the top and bottom walls. Periodic boundary conditions are imposed in the $x$ direction. The lateral alignment (in the $x$ direction) of the walls is specified by the register $\alpha$, which is defined by

$$
x_{i}^{u}=x_{i}^{l}+\alpha a,
$$

where $u$ and $l$, respectively, denote $x$ coordinates of corresponding atoms in the top and bottom walls and $\alpha$ is the fraction of the lattice constant by which the top wall is displaced laterally with respect to the bottom wall. In Fig. 1 the walls are shown precisely in register $(\alpha=0)$.

We assume that the configurational (potential) energy $U$ can be expressed as a sum of interactions between pairs of atoms

$$
\begin{aligned}
U\left(\mathbf{r}^{N_{f}}, \mathbf{r}^{N_{s}}\right)= & \frac{1}{2} \sum_{i=1}^{N_{f}} \sum_{j \neq i}^{N_{f}} u_{f f}\left(r_{i j}\right)+\sum_{i=1}^{N_{f}} \sum_{j=1}^{N_{s}} u_{f s}\left(r_{i j}\right) \\
& +\frac{1}{2} \sum_{i=1}^{N_{s}} \sum_{j \neq i}^{N_{s}} u_{s s}\left(r_{i j}\right)+\sum_{i=1}^{N_{s}} \sum_{j=1}^{N_{w}} u_{s s}\left(r_{i j}\right) .
\end{aligned}
$$

In Eq. (2) $\mathbf{r}^{N_{f}}$ and $\mathbf{r}^{N_{s}}$ stand for the collections of $2 N_{f}$ fluidatom coordinates and $2 N_{s}$ solid-atom coordinates, not including the solid atoms in the walls. Note that $U$ depends on the $2 N_{w}$ coordinates of the wall atoms through the last term in Eq. (2). We take the pair interactions to be shifted Lennard-Jones $(12,6)$ potentials

$$
u_{a b}(r)= \begin{cases}\phi_{a b}(r)-\phi_{a b}\left(r_{c}\right), & \text { if } r<r_{c} \\ 0, & \text { if } r \geqslant r_{c},\end{cases}
$$

where

$$
\phi_{a b}(r)=4 \epsilon_{a b}\left[(\sigma / r)^{12}-(\sigma / r)^{6}\right], \quad a b=f f, f s, s s .
$$

The effective diameter $\sigma$ and range $r_{c}$ are the same for all pairs; only the depth $\epsilon_{a b}$ of the attractive well depends on the composition of the pair. Thus, $U$ depends on only these parameters: $\boldsymbol{\epsilon}_{f f}, \boldsymbol{\epsilon}_{f s}, \boldsymbol{\epsilon}_{s s}$.

\section{HYBRID ATOMISTIC-COARSE-GRAINED DESCRIPTION}

Figure 1(b) displays a schematic of the partial coarse graining: A portion of each substrate (i.e. the far region) is covered by a mesh of congruent equilateral triangles (in the reference configuration), whose nodes coincide with a subset of (active) solid and wall atoms. We assume that when an element $e$ is distorted by displacing its nodes, the lattice underlying $e$ is homogeneously deformed. Then the coordinates $r_{i \alpha}$ of underlying atom $i$ can be written in terms of nodal coordinates $\left(R_{k \alpha}\right)$ as

$$
r_{i \alpha}=\sum_{k=1}^{3} A_{i}(k) R_{k \alpha} / A_{e} ; \quad \alpha=x, y,
$$

where $k$ labels nodes, $A_{e}$ denotes the area of $e$ and $A_{i}(k)$ denotes the area of the inscribed triangle which has one side 
coinciding with the side of $e$ opposite node $k$ and the opposite vertex coinciding with the lattice site at $\left(r_{i x}, r_{i y}\right)$, that is, with the atom $i{ }^{17}$ From Eq. (5) we conclude that the positions of all atoms underlying $e$ are uniquely and completely determined by the positions of the nodes of $e$. Therefore, under the assumption that the underlying atoms move in lockstep with the nodes, the number of independent variables needed to specify the configuration of the underlying atoms is greatly reduced, specifically from $2 N_{a}^{e}$, where $N_{a}^{e}$ is the number of atoms under $e$, to 6 .

The contribution of the coarse-grained portions of the substrates to the configurational energy can be written

$$
V\left(\mathbf{R}^{N_{n}}\right)=\sum_{e=1}^{N_{e}} N_{a}^{e} \tilde{u}_{e},
$$

where $\mathbf{R}^{N_{n}}$ stands for the nodal configuration, $N_{e}$ for the number of elements, and $\tilde{u}_{e}$ for the configurational energy per atom. Assuming that all elements are "local" (i.e., $e$ is large enough to contain the "cut-off" circle described below), we can express $\tilde{u}_{e}$ as

$$
\tilde{u}_{e}=\frac{1}{2} \sum_{j} u_{s s}\left(r_{i j}\right),
$$

where $i$ denotes the "centroid" atom (i.e., the atom nearest the centroid of $e$ ) and $j$ labels atoms that lie within the cut-off circle of radius $r_{c}$ that is centered on $i$. In essence $\tilde{u}_{e}$ is the configurational energy per atom for an infinite crystal that possesses the configuration of the homogeneous lattice underlying $e$. The total configurational energy of the partially coarse-grained system is then given by

$$
\begin{aligned}
U_{c}\left(\mathbf{r}^{N_{f}}, \mathbf{r}^{N_{s}^{\prime}}, \mathbf{R}^{N_{n}}\right)= & \frac{1}{2} \sum_{i=1}^{N_{f}} \sum_{j \neq i}^{N_{f}} u_{f f}\left(r_{i j}\right)+\sum_{i=1}^{N_{f}} \sum_{j=1}^{N_{s}^{\prime}} u_{f s}\left(r_{i j}\right) \\
& +\frac{1}{2} \sum_{i=1}^{N_{s}^{\prime}} \sum_{j \neq i}^{N_{s}^{\prime}} u_{s s}\left(r_{i j}\right)+\frac{1}{2} \sum_{i=1}^{N_{s}^{\prime}} \sum_{j=1}^{N_{s}^{\prime \prime}} u_{s s}\left(r_{i j}\right) \\
& +\sum_{e=1}^{N_{e}} N_{a}^{e} \frac{1}{2} \sum_{j \neq i} u_{s s}\left(r_{i j}\right),
\end{aligned}
$$

where $N_{s}^{\prime}$ stands for the number of atoms in the near regions and $N_{s}^{\prime \prime}$ for the number of atoms in the far (coarse-grained) regions. The second term in Eq. (8) assumes that the bands of near-region atoms are sufficiently wide that fluid atoms do not interact with underlying atoms in the far regions. The factor of $\frac{1}{2}$ in the next to last term in Eq. (8) prevents doubly counting the interactions between atoms in the near and far regions. The coarse-grained contribution (last term) effectively accounts for one half of the interactions between underlying and near-region atoms.

\section{STATISTICAL THERMODYNAMIC ANALYSIS}

We wish to study reversible shearing of the film under conditions of a constant number of fluid atoms $N_{f}$, constant (absolute) temperature $T$ and constant "normal" stress $T_{y y}$. For this purpose, we employ the analogue of the bulk-phase isothermal-isobaric ensemble, whose characteristic function $G$ is the analogue of the bulk-phase Gibbs energy:

$$
G=E-T S-L_{x} L_{y} T_{y y} .
$$

In Eq. (9) $E$ stands for the internal energy and $S$ for the entropy. The change in $G$ under a reversible transformation of the system is given by

$d G=-S d T+\mu d N_{f}+\gamma d L_{x}-L_{x} L_{y} d T_{y y}+T_{y x} L_{x} d(\alpha a)$,

where $\mu$ is the chemical potential of the fluid, $\gamma$ is the interfacial tension, and $T_{y x}$ is the shear stress, which is equivalent to the force per unit length acting upon the top wall in the $x$ direction. It is the shear stress upon which we wish to focus our attention primarily.

By means of a standard statistical-mechanical analysis we can derive the following formal relationship:

$$
G=-k_{B} T \ln \Delta,
$$

where $k_{B}$ is Boltzmann's constant and $\Delta$ is the isothermalisobaric partition function

$$
\Delta\left(T, N_{f}, T_{y y}, \alpha a\right)=\sum_{L_{y}} \exp \left[\left(L_{x} L_{y}\right) T_{y y} / k_{B} T\right] Q_{N_{f}} .
$$

In Eq. (12) $Q_{N_{f}}$ is the canonical partition function, which is expressible in the classical limit as

$$
\begin{aligned}
Q_{N_{f}}= & \frac{1}{h^{2\left(N_{f}+N_{s}\right)}} \frac{1}{N_{f} !} \int d \mathbf{p}^{N_{f}} \int d \mathbf{r}^{N_{f}} \int d \mathbf{p}^{N_{s}} \int d \mathbf{r}^{N_{s}} \\
& \times \exp \left(-H / k_{B} T\right),
\end{aligned}
$$

where $h$ stands for Planck's constant, the Hamiltonian $H$ is

$$
H=\sum_{i=1}^{N_{f}} \mathbf{p}_{i}^{2} / 2 m_{f}+\sum_{j=1}^{N_{s}} \mathbf{p}_{i}^{2} / 2 m_{s}+U\left(\mathbf{r}^{N_{f}}, \mathbf{r}^{N_{s}}\right),
$$

and $\mathbf{p}^{N_{f}}$ and $\mathbf{p}^{N_{s}}$ represent the momenta conjugate to the coordinates $\mathbf{r}^{N_{f}}$ and $\mathbf{r}^{N_{s}}$, respectively.

From Eqs. (10)-(13) we obtain for the shear stress

$$
\begin{aligned}
L_{x} T_{y x}=\frac{\partial G}{\partial \alpha a} & =-\frac{k_{B} T}{\Delta} \frac{\partial \Delta}{\partial \alpha a} \\
& =-k_{B} T \frac{\sum_{L_{y}} \exp \left(L_{x} L_{y} T_{y y} / k_{B} T\right) \frac{\partial Z_{N_{f}}}{\partial \alpha a}}{\sum_{L_{y}} \exp \left(L_{x} L_{y} T_{y y} / k_{B} T\right) Z_{N_{f}}},
\end{aligned}
$$

where the configuration integral $Z_{N_{f}}$ is

$$
Z_{N_{f}}=\int d \mathbf{r}^{N_{f}} \int d \mathbf{r}^{N_{s}} \exp \left(-U / k_{B} T\right) .
$$

The second line of Eq. (15) follows from the observation that the integrals on momenta do not depend on $L_{y}$ or $\alpha$. Employing procedures detailed previously, ${ }^{19,20}$ we derive the following formula for the shear stress 


$$
\begin{aligned}
T_{y x}= & \frac{1}{2 L_{x}} \sum_{i=1}^{N_{f}} \sum_{j \neq i}^{N_{f}}\left\langle u_{f f}^{\prime}\left(r_{i j}\right) x_{i j} y_{i j} /\left(r_{i j} L_{y}\right)\right\rangle \\
& +\frac{1}{L_{x}} \sum_{i=1}^{N_{f}} \sum_{j=1}^{N_{s}}\left\langle u_{f s}^{\prime}\left(r_{i j}\right) x_{i j} y_{i j} /\left(r_{i j} L_{y}\right)\right\rangle \\
& +\frac{1}{2 L_{x}} \sum_{i=1}^{N_{s}} \sum_{j \neq i}^{N_{s}}\left\langle u_{s s}^{\prime}\left(r_{i j}\right) x_{i j} y_{i j} /\left(r_{i j} L_{y}\right)\right\rangle \\
& +\frac{1}{L_{x}} \sum_{i=1}^{N_{s}} \sum_{j=1}^{N_{w}}\left\langle u_{s s}^{\prime}\left(r_{i j}\right) x_{i j} y_{i j} /\left(r_{i j} L_{y}\right)\right\rangle .
\end{aligned}
$$

Here the brackets signify the ensemble average; the prime denotes the derivative with respect to the argument of the function. Equation (17) is employed in the Monte Carlo simulation of the fully atomistic system, as described in Sec. V.

The hybrid analogue of Eq. (14) is

$$
\begin{aligned}
H_{c}= & \sum_{i=1}^{N_{f}} \mathbf{p}_{i}^{2} / 2 m_{f}+\sum_{i=1}^{N_{s}^{\prime}} \mathbf{p}_{i}^{2} / 2 m_{s}+\frac{1}{2} \mathbf{P}^{T}(\mathbf{M})^{-1} \mathbf{P} \\
& +U_{c}\left(\mathbf{r}^{N_{f}}, \mathbf{r}^{N_{s}^{\prime}}, \mathbf{R}^{N_{n}}\right),
\end{aligned}
$$

where $\mathbf{P}$ is the $2 N_{n}$-dimensional column vector of momenta conjugate to the nodal coordinates $\mathbf{R}^{N_{n}}$ and $\mathbf{M}^{-1}$ is the effective mass matrix, which depends on the transformation Eq. (5). ${ }^{18}$ By a derivation that parallels the one reaching Eq. (17) we obtain the hybrid analogue of Eq. (17)

$$
\begin{aligned}
T_{y x, c}= & \frac{1}{2 L_{x}} \sum_{i=1}^{N_{f}} \sum_{j \neq i}^{N_{f}}\left\langle u_{f f}^{\prime}\left(r_{i j}\right) x_{i j} y_{i j} /\left(r_{i j} L_{y}\right)\right\rangle \\
& +\frac{1}{L_{x}} \sum_{i=1}^{N_{f}} \sum_{j=1}^{N_{s}^{\prime}}\left\langle u_{f s}^{\prime}\left(r_{i j}\right) x_{i j} y_{i j} /\left(r_{i j} L_{y}\right)\right\rangle \\
& +\frac{1}{2 L_{x}} \sum_{i=1}^{N_{s}^{\prime}} \sum_{j \neq i}^{N_{s}^{\prime}}\left\langle u_{s s}^{\prime}\left(r_{i j}\right) x_{i j} y_{i j} /\left(r_{i j} L_{y}\right)\right\rangle \\
& +\frac{1}{2 L_{x}} \sum_{i=1}^{N_{s}^{\prime}} \sum_{j=1}^{N_{s}^{\prime \prime}}\left\langle u_{s s}^{\prime}\left(r_{i j}\right) x_{i j} y_{i j} /\left(r_{i j} L_{y}\right)\right\rangle \\
& +\frac{1}{L_{x}} \sum_{e=1}^{N_{e}} N_{a}^{e}\left[\frac{1}{2} \sum_{j \neq i}\left\langle u_{s s}^{\prime}\left(r_{i j}\right) x_{i j} y_{i j} /\left(r_{i j} L_{y}\right)\right\rangle\right]
\end{aligned}
$$

where $i$ in the last term refers to the centroid atom.

\section{COMPUTATIONAL PROCEDURE}

Figure 1 displays schematic diagrams of the initial state of the system for the "exact" (i.e., fully atomistic) and several approximate versions of the idealized contact. The walls are in register $(\alpha=0)$ and each substrate has the configuration of the perfect crystal. The separation $L_{y}$ between the walls is such that a monolayer of fluid atoms just fits in the interstices formed by the solid surface atoms. The initial configuration of fluid atoms is random.
To compute the thermomechanical properties we perform isothermal-isobaric Monte Carlo (MC) simulations using the prescription given previously. ${ }^{19,20}$ One MC cycle consists of a sequence of diffusive steps (i.e., attempted displacements of atoms or nodes with $L_{y}$ fixed) followed by a homogeneous compression or dilation of the system in the $y$ direction. The diffusive part of the cycle comprises two subsequences. In the first atoms (or nodes) are sequentially displaced just once at random (i.e., the atom or node is randomly displaced within a square of side $\delta$ centered on its initial position). In the second subsequence, fluid atoms are subjected to several cycles of "large" moves in order to facilitate the equilibration of the fluid film, which tends to get trapped in metastable configurations. During one cycle of large moves the $N_{f}$ fluid atoms are sequentially displaced randomly in a square of edge $\Delta>\delta$. We generate a total of $M$ $\mathrm{MC}$ cycles, discard the first half of these, and evaluate ensemble averages over the remaining half.

Shearing, which is regarded here as a quasistatic (reversible) process, is effected by increasing $\alpha$ gradually in small increments $\Delta \alpha$. The initial configuration for any register $(\alpha \neq 0)$ is determined from the last configuration of the previous register $(\alpha-\Delta \alpha)$. In the absence of coarse-graining the previous $x$ coordinates of atoms in the $n$ rows nearest the top wall (or nearest the plane of the rigid substrate bordering the interfacial region) are displaced by

$$
\Delta x(y)=a\left(1-\frac{y_{w}-y}{n b}\right) \Delta \alpha,
$$

where $b$ is the mean distance between lines of atoms parallel with the $x$ axis, $y_{w}$ is the common $y$ coordinate of the wall atoms (or atoms in the nearest plane of rigid substrate) and $y$ designates the coordinate of the solid atom. In case the solid is partly coarse-grained, then the line of nodes in the top wall is displaced by $\Delta x=a \Delta \alpha$, but the active nodes are assumed to remain fixed. Hence, underlying atoms are displaced from their positions in the previous configuration according to Eq. (5). For each successive register we repeat the MC simulation, as described above for the case $\alpha=0$.

For all simulations, Table I lists the values of the various parameters. Numerical values are expressed in dimensionless units based on the Lennard-Jones parameters for the solidsolid interaction: Distance is expressed in units of $\sigma$; energy in units of $\epsilon_{s s}$; stress in units of $\epsilon_{s s} / \sigma$; temperature in units of $\epsilon_{s s} / k_{B}$.

\section{RESULTS}

\section{A. Fully atomistic treatment}

We first survey the behavior of the fully atomistic version [see Fig. 1(a)] of the two-dimensional (2D) contact under reversible shearing. Figure 2 displays plots of the shear stress $T_{y x}$ as a function of register $\alpha$. The two curves correspond to shearing in the "forward" $(\alpha=0 \rightarrow \alpha=1.2)$ and "reverse" ( $\alpha=1.2 \rightarrow \alpha=0)$ directions. Note that since shearing is a quasistatic process (i.e., it is carried out infinitely slowly), each point $\left(\alpha, T_{y x}\right)$ of the shear-stress curve corresponds to an equilibrium state of the system. The plots are periodic in $\alpha$, having a period of unity. They are also antisymmetric 
TABLE I. Parameters (in dimensionless units defined in text) of the simulation.

$N_{f}=10$
$N_{w}=2 \times 10=20$
$N_{s}=2 \times 240=480$
$N_{s}^{\prime}=2 \times 40=80$
$N_{s}^{\prime \prime}=2 \times 200=400$
$N_{e}=2 \times 4=8$
$N_{n}=2 \times 2=4$
$T=0.1$
$T_{y y}=-0.1$
$\sqrt{\frac{2}{\sqrt{3}}}=1.075$
$L_{x}=10 a$
$\epsilon_{s s}=1$
$\epsilon_{f f}=1 / 9$
$\epsilon_{s f}=1 / 9$
$r_{c}=2.5$
$M=10^{6}$

around $\alpha=0.5$ in the range $0<\alpha<1.0$. The maximum in $T_{y x}$, which occurs around $\alpha \approx 0.4$, is the minimum stress needed to initiate sliding of the contact in the forward direction. Hence the static friction force per unit length of the contact can be taken to be the maximum in $T_{y x}$.

The symmetry of the shear-stress curve can be rationalized as follows. The explanation is based largely on snapshots of atomic configurations, which we do not present for lack of space. When $\alpha=0$, the solid surfaces at the fluidsolid interface are aligned so that the solid atoms in the upper surface are precisely opposite (on average) those in the lower surface. This configuration of the solid atoms at the interface has the effect of creating interstices (cages) into which the fluid atoms neatly fit. The mean position of a given fluid atom is at the center of its cage. It thus exerts equal and opposite forces on the two atoms of the upper solid surface, as well as on the two atoms of the lower surface, which altogether form its cage. Thus, the net transverse ( $x$ direction) force on the upper substrate vanishes at $\alpha=0$. We note that if the fluid atoms were replaced by solid atoms, then the system would become a pure single crystal under no shear stress.

Now as the top wall is displaced slightly to the right $(\alpha=0 \rightarrow \alpha=\Delta \alpha)$, that is, as the system is subjected to a small shear strain, the atoms relax upon equilibration (after many MC cycles starting from the initial configuration) to their new equilibrium positions, which are shifted to the right. In particular, the atoms in the upper solid surface at the interface are shifted to the right of their counter-atoms in the lower solid surface. As a consequence, the mean position of a fluid atom in its cage is closer to the upper (lower) solid atom that lies to its left (right). Hence, the fluid atoms exert a net transverse force (in the negative $x$ direction) on the upper solid surface, which is transmitted to the top wall and must be counterbalanced by an external force applied in the positive $x$ direction in order to hold the wall in place (i.e., to fix the value of $\alpha=\Delta \alpha$ ). The applied external force per unit length is the shear stress. By convention, the sign of $T_{y x}$ is positive if the applied force is in the positive $x$ direction.

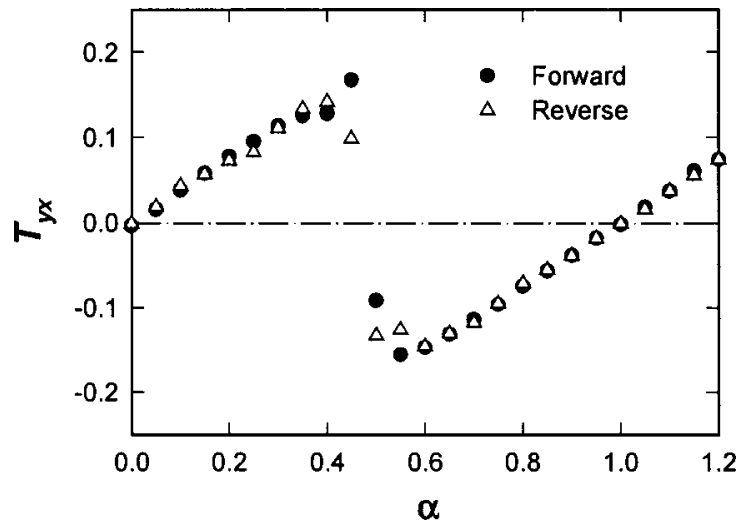

FIG. 2. Shear-stress curves (shear stress $T_{y x}$ vs register $\alpha$ ) for the fully atomistic version [Fig. 1(a)] of the 2D model contact.

As $\alpha$ increases gradually over the range $0<\alpha \leqslant 0.4$ and the effective cages of the fluid atoms become more constricted, $T_{y x}$ rises (Fig. 2). The system behaves as if it were a linear elastic solid. When the register just exceeds $\alpha \simeq 0.4$, $T_{y x}$ begins to drop precipitously. The configuration snapshots indicate that the atoms of the upper solid surface at the interface abruptly slip over the caged fluid atoms, so that they (the upper solid atoms) lie nearly over the voids between the atoms in the lower solid surface. In this situation the old cages shrink to create new ones. There are about twice as many cages of about half the size. In the range $0.4 \leqslant \alpha<0.5$, the newly created cages are smaller than the shrunken original ones, so that (on average) fluid molecules are asymmetrically distributed about atoms in the upper solid surface. Hence, there remains a net transverse force on the upper wall, although it is diminished substantially from the maximum at $\alpha \simeq 0.4$, where fluid atoms are constrained to the original cages. Finally, at $\alpha=0.5$ the cages are of the same size. Fluid atoms should, therefore, be symmetrically distributed about solid atoms in the upper surface so that the net transverse force on the upper wall vanishes.

As $\alpha$ varies over the range $0.5<\alpha<1.0$, the sequence of events detailed above is reversed, except that the distribution of fluid atoms is skewed in the opposite direction. As a consequence the net transverse force on the top wall changes sign. When the register finally reaches $\alpha=1$, the system is in precisely the same condition as it is at $\alpha=0$. Thus, as $\alpha$ increases from 1.0, the above scenario is repeated.

Shearing from $\alpha=1.2$ to $\alpha=0$ carries the system in reverse through precisely the same states as shearing in the forward direction. Since the process is performed reversibly, we expect the "reverse" plot to coincide with the "forward" one, as indicated in Fig. 2.

We regard the version of the model contact depicted in Fig. 1(a) as a prototype and the results of the fully atomistic simulation (Fig. 2) as "exact." They furnish a benchmark against which we can compare results of simulations of approximate versions, which we consider next.

\section{B. Approximate dynamical treatment of substrates}

Is it necessary to describe the entire system at the atomistic scale in order to obtain reliable shear-stress curves (i.e., 


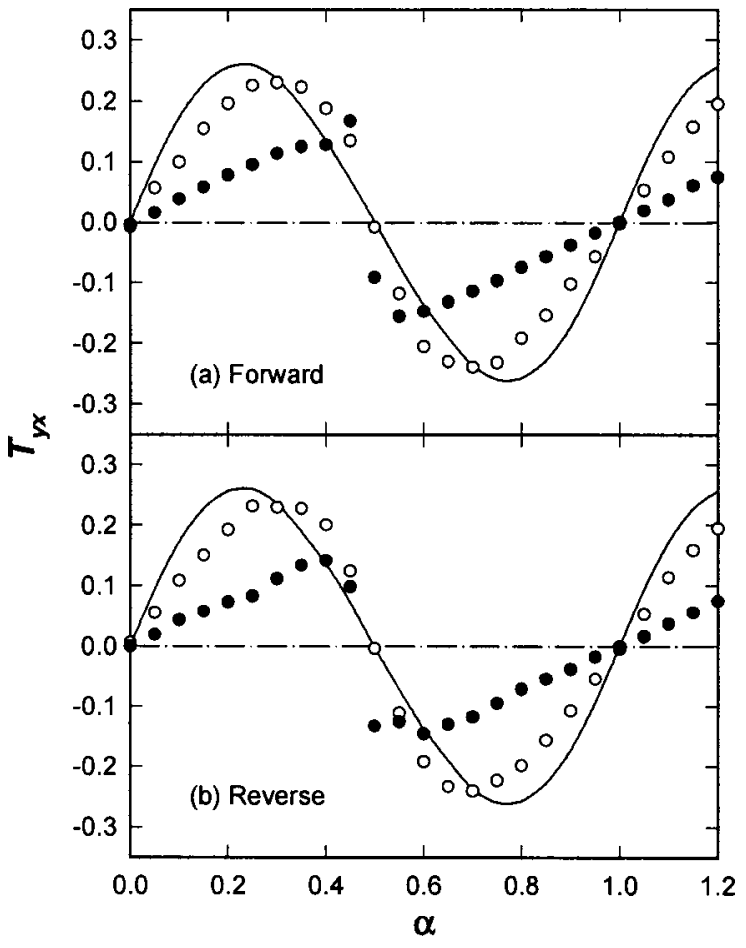

FIG. 3. Comparison of shear-stress curves for several versions of 2D (model) contact: Wholly rigid substrates (solid lines) [Fig. 1(c)]; only rigid far regions (open circles) [Fig. 1(d)]; fully atomistic (solid circles) [Fig. $1(\mathrm{a})]$.

curves that essentially agree with those displayed in Fig. 2)? If so, then we face an overwhelming computational burden in treating general tribological systems, which involve billions of atoms. (Even our relatively small model contact, which is deliberately tailored for our present purpose, taxes our computational resources when it becomes necessary to simulate a large number of thermodynamic states.) Since we are convinced of the necessity of describing the film and near regions at the atomic level, our only hope is that the dynamic response of the far regions to molecular motion in the film and near regions can be handled approximately in such a way as to yield reliable shear-stress curves.

We can conceive of several levels of approximation that could be adequate to this task. At the crudest level we simply freeze the entire solid substrate, as shown in Fig. 1(c). That is, we take the substrates to be wholly rigid. We note in passing that this is a common approximation..$^{5-7}$ The rigid substrate can be regarded as an extended wall. Only the first two terms on the right side of Eq. (17) contribute to the shear stress and $L_{y}$ refers to the separation between the planes of the rigid substrates that border the film. Figure 3 displays the resulting shear-stress curve. Although the curve exhibits the same symmetries (periodic in $\alpha$ of period unity; antisymmetric about $\alpha=0.5$ in the range $0<\alpha<1$ ) as the exact curve (Fig. 2), which can be rationalized in a similar way, it is not even qualitatively correct in that it fails to reproduce the "snapping" character of the exact curve (i.e., the slow rise with register in the elastic region followed by the precipitous decline at the threshold of the transition region, which suggests a tendency of the contact to lurch at a critical register).

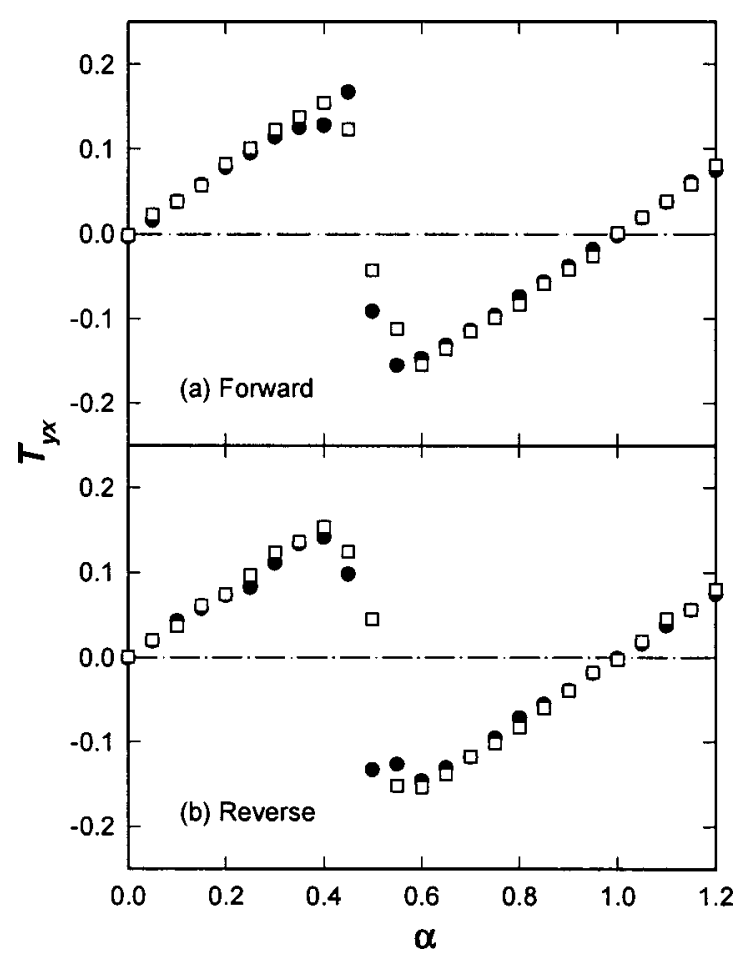

FIG. 4. Comparison of shear-stress curves for the fully atomistic (solid circles) [Fig. 1(a)] and hybrid (open squares) [Fig. 1(b)] versions of the 2D model contact.

The next level of approximation that suggests itself is to improve the crudest model by "thawing" the near region of the substrates [Fig. 1(d)]. As Fig. 3 indicates, the introduction of a measure of elasticity in the substrates improves the shape of the shear-stress curve modestly by lowering the maximum and shifting it to higher register. Nevertheless, the approximate curve still does not capture the snapping character of the exact one.

We could of course extend the atomistically treated near regions indefinitely, eventually reaching the exact limit [Fig. 1(a)], but this tack would obviously defeat our objective of finding an efficient and reliable method of treating the far regions approximately. Therefore, we attempt to introduce elasticity at the continuum scale by coarse-graining the far regions [Fig. 1(b)]. As illustrated in Fig. 1(b), we take (the equivalent of) four triangles in the mesh on each substrate. On account of the periodic boundary conditions in the $x$ direction there are two active nodes in each substrate (i.e., nodes coinciding with solid atoms). These are sufficient to determine the positions of 200 (active) underlying atoms. Plots of coarse-grained and exact shear-stress curves in Fig. 4 are in excellent agreement, thus showing that coarsegraining can reliably approximate the elastic response of the far regions.

\section{SUMMARY AND CONCLUSIONS}

We present a new hybrid atomistic coarse-grained treatment of thin-film lubrication. The key idea is to coarse-grain the regions of the solid substrates that are sufficiently far removed from the lubricant (fluid)-solid interface. The coarse-graining, which is accomplished by superposing a 
mesh on the far regions, reduces the original Hamiltonian to an effective Hamiltonian which governs the motion of the original fluid atoms plus the near-region solid atoms plus the nodes of the mesh as pseudo-atoms. The effective Hamiltonian can be used to carry out Monte Carlo simulations of (reversible) sliding.

We applied the new hybrid atomistic-coarse-grained scheme to an ideal 2D lubricated contact consisting of a monolayer of fluid between two planar solid substrates. Simulating reversible shearing of the contact by means of an isobaric-isothermal ensemble Monte Carlo method, we studied several approximate versions of the model designed to ascertain the importance of a proper accounting for the elastic response of the substrates. The conclusions of the investigation are:

(1) Neglecting completely the elastic response of the substrates (i.e., maintaining the substrates entirely rigid), one misses completely the "snapping" character of the exact shear-stress curve (i.e., the gradual rise of the stress with register followed by an abrupt drop at the threshold of the transition region, suggesting a tendency of the contact to snap at a critical register);

(2) introducing elasticity into the near regions of the substrates, while maintaining the far regions rigid, improves the shape of the shear-stress curves only marginally, which suggests that the elastic response of the far regions is critical to a reliable overall description;

(3) the elastic response of the far regions can be adequately and efficiently accounted for by the hybrid atomisticcoarse-graining scheme.

\section{ACKNOWLEDGMENTS}

We thank the National Science Foundation for generous support through the Grant Programs ECS-9900127, ITR0112929, and NIRT-0210850. We also thank the UNL research computing facility for support.

${ }^{1}$ N. P. Suh, Tribophysics (Prentice-Hall, Inglewood Cliffs, NJ, 1986).

${ }^{2}$ F. P. Bowden and D. Tabor, Friction and Lubrication of Solids (Clarendon, Oxford, 1964).

${ }^{3}$ D. H. Buckley, Surface Effects in Adhesion, Friction, Wear and Lubrication (Elsevier, Amsterdam, 1981).

${ }^{4}$ D. Godfery, in Fundamentals of Tribology, edited by N. P. Suh and H. Saka (MIT Press, Cambridge, 1980).

${ }^{5}$ B. Bhushan, J. N. Israelachvili, and U. Landman, Nature (London) 374, 607 (1995).

${ }^{6}$ M. H. Müser, M. Urbakh, and M. O. Robbins, Adv. Chem. Phys. 126, 187 (2003).

${ }^{7}$ M. O. Robbins and M. H. Müser, in Modern Tribology Handbook, Vol. 1, edited by B. Bhushan (CRC Press, Boca Raton, 2001), p. 717.

${ }^{8}$ J. Baschnagel, K. Binder, P. Doruker et al., Adv. Polym. Sci. 152, 41 (2000).

${ }^{9}$ C. N. Likos, Phys. Rep. 348, 267 (2001).

${ }^{10}$ F. Müller-Plathe, ChemPhysChem 3, 754 (2002).

${ }^{11}$ R. E. Rudd and J. Q. Broughton, Phys. Status Solidi B 217, 251 (2000).

${ }^{12}$ R. E. Miller and E. B. Tadmor, J. Comput.-Aided Mater. Des. 9, 206 (2002).

${ }^{13}$ S. Glotzer and W. Paul, Annu. Rev. Mater. Sci. 32, 401 (2002).

${ }^{14}$ R. M. Nieminen, J. Phys.: Condens. Matter 14, 2859 (2002).

${ }^{15}$ R. Phillips, M. Dittrich, and K. Schulten, Annu. Rev. Mater. Sci. 32, 219 (2002).

${ }^{16}$ E. B. Tadmor, M. Ortiz, and R. Phillips, Philos. Mag. A 73, 1529 (1996).

${ }^{17}$ O. C. Zienkiewicz and R. L. Taylor, The Finite Element Method, 5th ed. (Butterworth-Heinemann, Oxford, 2000), Vol. 1, Chap. 4.

${ }^{18}$ Z.-B. Wu, D. J. Diestler, R. Feng, and X. C. Zeng, J. Chem. Phys. 119, 8013 (2003).

${ }^{19}$ M. Schoen, D. J. Diestler, and J. H. Cushman, Phys. Rev. B 47, 5603 (1993).

${ }^{20}$ M. Schoen, S. Hess, and D. J. Diestler, Phys. Rev. E 52, 2587 (1995). 